\title{
Universality of the EPR-chameleon model
}

\author{
Luigi Accardi \\ Centro Vito Volterra \\ Università degli Studi di Roma "Tor Vergata \\ Via Columbia, Rome 00133, Italy \\ accardi@volterra.uniroma2.it \\ Satoshi Uchiyama \\ Department of Life and Creative Sciences \\ Hokusei Gakuen University Junior College \\ Atsubetsu-ku, Sapporo 004-8631, Japan \\ uchiyama@hokusei.ac.jp
}




\section{Indice}

1 introduction 3

1.1 The chameleon effect . . . . . . . . . . . 4

2 The EPR-chameleon dynamical system 5

$\begin{array}{lll}3 & \text { The uniquenenss theorem } & 7\end{array}$

4 Local, causal measurements $\quad 14$

5 Trivial extremal LC probability measures 18

6 Bell implicitly assumes triviality 23

7 Empirical Correlations of pairs of distant particles $\quad 25$

7.1 Standard correlations . . . . . . . . . . . 25

7.2 Correlations of distant pairs . . . . . . . . . 27 


\begin{abstract}
In terms of EPR-chameleon models and local and causal measures, the Bell's argument is reanalyzed. Contrary to Bell, it is shown that the nontriviality of the joint probability measure does not always imply the nonlocality. It is analyzed that under what conditions correlations of distant particles are obtained which are different from the standard correlations. The protocol for the correlations of distant particles admits nontrivial probability measures respecting the locality.
\end{abstract}

\title{
1 introduction
}

In the first section of this paper we briefly recall the basic physical idea of the chameleon effect (i.e. the theory of adaptive dynamical systems) and, in the second one, the main theorem on the reversible, deterministic model constructed in $[1,2]$ (hereinafter EPR-chameleon model), which reproduces the EPRBohm correlations in full respect of causality and locality. Furthermore, as it is apparent from the statement of Theorem (1) below, there is no artificial post-selection in the assumptions of the theorem (no "cospiracy of the detectors").

In the third section we discuss the recent result (see [3]) that the EPR-chameleon model is the only possible model with this property, in a sense that will be specified in the following.

In the EPR-chameleon model, a 'configuration' of the system changes during the measurement process. This change is done locally: action at a distance in the measurement process is unnecessary.

On the other hand, according to a widespread belief, Bell's argument implies that there is no local hidden variable theory that reproduces the EPR-Bohm correlations. 
This belief however is based on some assumptions, which were implicit in Bell's argument and which have remained implicit for a long time. The quantum probability approach, started about 30 years ago, has made these assumptions explicit thus introducing, in the debate on the foundations of quantum theory, two main new ingredients, namely:

(i) the chameleon effect, as an intuitive explanation of the mechanism through which non Kolmogorovian statistics can be produced by classical deterministic systems

(ii) the difference between trivial and non-trivial local measures.

Statement (i), which implies that the meaning of the Bell inequality consists in the proof of the existence of sets of experimentally measurable statistical data, coming from similar but incompatible experiments, which cannot be described by a single Kolmogorov model, is nowadays widely accepted in the literature.

Therefore in the present paper we will concentrate our attention on statement (ii), which has a more subtle mathematical nature. This will be done starting from the fourth section below.

\subsection{The chameleon effect}

The chameleon effect gives a mathematically precise formulation to the widely used (and abused) statement that a measurement determines the value of an observable: it distinguishes those measurements which register a previously existing property, independent of the measurement itself, from the adaptive measurements, which register the reaction to a given interaction. 
The measurement of the color of a billiard ball provides a good metaphor of the former situation. The measurement of the color of the skin of a chameleon - of the latter.

We speak of chameleon effect whenever the dynamics of a system depends on the observable that one measures. This property can be taken as definition of adaptive dynamical systems.

Both situations are compatible with EPR's requirement of pre-determination, but in the former case this term should be interpreted as the passive reading of a pre-existing property; in the latter - as the active determination of a pre-established response.

The two interpretations are reflected in two different mathematical models and, in the following, we are going to illustrate these differences.

\section{The EPR-chameleon dynamical system}

In this section we briefly recall the EPR-chameleon theorem, proved in [1] which gives in particular a classical, deterministic, reversible, local dynamical system that reproduces exactly the EPR correlations.

Theorem 1 (i) For $a, b \in[0,2 \pi)$ the maps (local adaptive dynamics)

$$
\begin{gathered}
T_{1, a}, T_{2, b}:[0,2 \pi) \times \mathbf{R} \rightarrow[\mathbf{0}, \mathbf{2} \pi) \times \mathbf{R} \\
T_{1, a}\left(\sigma_{1}, \lambda_{1}\right):=\left(\sigma_{1}, \frac{\sqrt{2 \pi}\left|\cos \left(\sigma_{1}-a\right)\right| \lambda_{1}}{4}\right) \in[0,2 \pi) \times \mathbf{R} \\
T_{2, b}\left(\sigma_{2}, \lambda_{2}\right):=\left(\sigma_{2}, \sqrt{2 \pi} \lambda_{2}\right) \in[0,2 \pi) \times \mathbf{R}
\end{gathered}
$$


are invertible with inverses:

$$
\begin{gathered}
T_{1, a}^{-1}\left(\sigma_{1}, \lambda_{1}\right):=\left(\sigma_{1}, \frac{4 \lambda_{1}}{\sqrt{2 \pi}\left|\cos \left(\sigma_{1}-a\right)\right|}\right) \\
T_{2, b}^{-1}\left(\sigma_{2}, \lambda_{2}\right):=\left(\sigma_{2}, \frac{\lambda_{2}}{\sqrt{2 \pi}}\right)
\end{gathered}
$$

(ii) Denote $\hat{T}_{j, x}^{-1}$ the second component of $T_{j, x}^{-1}(j=1,2, x=$ $a, b)$ and $m_{a}, m_{b}$ arbitrary real numbers. Then the following positive measure on $[0,2 \pi)^{2} \times \mathbf{R}^{\mathbf{2}}$ (initial distribution) is well defined and is a probability measure:

$P_{a, b}\left(d \sigma_{1} d \sigma_{2} d \lambda_{1} d \lambda_{2}\right):=p_{S}\left(\sigma_{1}, \sigma_{2}\right) p_{1, a}\left(\sigma_{1}, \lambda_{1}\right) p_{2, b}\left(\sigma_{2}, \lambda_{2}\right) d \sigma_{1} d \sigma_{2} d \lambda_{1} d \lambda_{2}:=$

$:=\frac{1}{2 \pi} \delta\left(\sigma_{1}-\sigma_{2}\right) d \sigma_{1} d \sigma_{2} \delta\left(\hat{T}_{1, a}^{-1}\left(\sigma_{1}, \lambda_{1}\right)-m_{a}\right) d \lambda_{1} \delta\left(\hat{T}_{2, b}^{-1}\left(\sigma_{2}, \lambda_{2}\right)-m_{b}\right) d \lambda_{2}$

(iii) The $P_{a, b}$-pair correlations of the \pm 1 -valued observables

$$
\begin{gathered}
S_{a}^{(1)}, S_{b}^{(2)}:[0,2 \pi) \times \mathbf{R} \rightarrow\{ \pm \mathbf{1}\} \\
S_{a}^{(1)}(\sigma, \mu):=\operatorname{sgn}(\cos (\sigma-a)) \\
S_{b}^{(2)}(\sigma, \mu):=-\operatorname{sgn}(\cos (\sigma-b))=-S_{b}^{(1)}(\sigma, \mu)
\end{gathered}
$$

reproduce the EPR correlations for any value of the parameters $m_{a}, m_{b}$, i.e.

$$
\int S_{a}^{(1)}\left(\sigma_{1}, \lambda_{1}\right) S_{b}^{(2)}\left(\sigma_{2}, \lambda_{2}\right) P_{a, b}\left(d \sigma_{1} d \sigma_{2} d \lambda_{1} d \lambda_{2}\right)=-\cos (a-b)
$$

\section{Remark .}

The physical meaning of the inverse maps $\hat{T}_{j, x}^{-1}$ in the definition of the initial distribution (6) is explained in the paper [4]. 


\section{The uniquenenss theorem}

In the present section we study the most general family of local causal probability measures which reproduce the EPR-Bohm correlations and we prove that, under natural generic conditions and up to convex combinations, they must have the form described in the EPR-chameleon model constructed in $[1,2]$.

We will use the following notations. The configuration space of the single particle is identified to the unit circle, i.e.

$S_{1}=S_{2}=S^{1}:=\left\{(x, y) \in \mathbf{R}^{2}: \mathbf{x}^{2}+\mathbf{y}^{2}=\mathbf{1}\right\} \equiv[\mathbf{0}, \mathbf{2} \pi) \equiv \mathbf{R} /(\mathbf{2} \pi \mathbf{Z})$

and the observables to periodic functions $f: \mathbf{R} \rightarrow \mathbf{R}$ with period $2 \pi$. We denote

$$
T^{2}:=S^{1} \times S^{1}
$$

and define the intervals

$$
\begin{aligned}
I_{a} & :=\left[-\frac{\pi}{2}+a, a+\frac{\pi}{2}\right), \\
J_{a} & :=\left[a+\frac{\pi}{2}, a+\frac{3 \pi}{2}\right)
\end{aligned}
$$

The random variables $S_{a}^{(1)}$ and $S_{b}^{(2)}(a, b \in[0,2 \pi))$ are defined by

$$
\begin{gathered}
S_{a}^{(1)}\left(s_{1}\right):=\chi_{I_{a}}\left(s_{1}\right)-\chi_{J_{a}}\left(s_{1}\right) \quad, s_{1} \in S_{1} \\
S_{b}^{(2)}\left(s_{2}\right):=-\chi_{I_{b}}\left(s_{2}\right)+\chi_{J_{b}}\left(s_{2}\right) \quad, s_{2} \in S_{2}
\end{gathered}
$$

If $P_{a, b}$ is a local causal probability measure on $S_{1} \times S_{2} \times M_{1} \times$ $M_{2}$ we denote $R_{a, b}$ its marginal probability on $T^{2}=S^{1} \times S^{1}$. Thus:

$$
d R_{a, b}\left(s_{1}, s_{2}\right)=d P_{S}\left(s_{1}, s_{2}\right) p_{1, a}\left(s_{1}\right) p_{2, b}\left(s_{2}\right)
$$

where $s_{1}, s_{2} \in[0,2 \pi)$ are fixed parameterizations of $S_{1}=S^{1}$ and $S_{2}=S^{1}$ respectively, $P_{S}$ is a probability measure on $T^{2}$ and $p_{1, a}\left(s_{1}\right), p_{2, b}\left(s_{2}\right) \geq 0$. 
Definition 1 A family $\left\{R_{a, b}: a, b \in[0,2 \pi)\right\}$ of probability measures is said to reproduce the EPR statistics if for any $a, b \in[0,2 \pi)$ one has:

$$
\begin{aligned}
& R_{a, b}\left(I_{a} \times I_{b}\right)=\frac{1}{2} \cos ^{2}\left(\frac{b-a}{2}\right)=: P_{a, b}^{+-} \\
& R_{a, b}\left(J_{a} \times J_{b}\right)=\frac{1}{2} \cos ^{2}\left(\frac{b-a}{2}\right)=: P_{a, b}^{-+} \\
& R_{a, b}\left(I_{a} \times J_{b}\right)=\frac{1}{2} \sin ^{2}\left(\frac{b-a}{2}\right)=: P_{a, b}^{++} \\
& R_{a, b}\left(J_{a} \times I_{b}\right)=\frac{1}{2} \sin ^{2}\left(\frac{b-a}{2}\right)=: P_{a, b}^{--}
\end{aligned}
$$

Remark (1). Notice the rotation invariance of the experimentally measured probabilities.

Remark (2). The class of all families $\mathcal{R}:=\left\{R_{a, b}: a, b \in\right.$ $[0,2 \pi)\}$, of probability measures which reproduce the EPR statistics, is a closed convex set if topology and convex combinations are defined as follows.

The sequence $\left(\mathcal{R}_{n}\right)$ is said to converge to $\mathcal{R}$ if, for every $a, b \in$ $[0,2 \pi)$ the sequence of probability mesures $\left(R_{a, b ; n}\right)$ converges weakly to the probability mesure $R_{a, b}$.

The family $\mathcal{R}$ is said to be a convex combination of the two families $\mathcal{P}, \mathcal{Q}$ if there exists $t \in[0,1]$ such that, for every $a, b \in$ $[0,2 \pi)$ one has (in obvious notations)

$$
R_{a, b}=t P_{a, b}+(1-t) Q_{a, b}
$$

Definition 2 The family of LC probability measures

$$
d R_{a, b}\left(s_{1}, s_{2}\right)=d P_{S}\left(s_{1}, s_{2}\right) p_{1, a}\left(s_{1}\right) p_{2, b}\left(s_{2}\right)
$$


is said to satisfy the condition of statistical pre-determination if $\forall\left(s_{1}, s_{2}\right) \in T^{2} \backslash \Delta$ there exists $a \in S^{1}$ and a neighborhood $G$ of $\left(s_{1}, s_{2}\right)$, contained in $\left(I_{a} \times J_{a}\right) \cup\left(J_{a} \times I_{a}\right)$ such that

$$
p_{1, a}\left(s_{1}^{\prime}\right) p_{2, a}\left(s_{2}^{\prime}\right)>0 \quad ; \quad \forall\left(s_{1}^{\prime}, s_{2}^{\prime}\right) \in G
$$

\section{Remark.}

Statistical predetermination means that, the fact that a configuration is statistically forbidden for all measurements such that the outcomes are precisely (anti-)correlated cannot depend on the local measurements, but it is defined at the source.

Theorem 2 Suppose that the family of probability measures

$$
d R_{a, b}\left(s_{1}, s_{2}\right)=d P_{S}\left(s_{1}, s_{2}\right) p_{1, a}\left(s_{1}\right) p_{2, b}\left(s_{2}\right)
$$

satisfies the conditions:

(i) reproduce the EPR statistics

(ii) statistical pre-determination.

Then the source distribution $P_{S}$ must have support in the diagonal of $T^{2}$

$$
\operatorname{supp}_{S} \subseteq \Delta:=\left\{\left(s_{1}, s_{2}\right) \in T^{2}: s_{1}=s_{2}(\bmod 2 \pi)\right\}
$$

If in addition

(iii) the restriction of $P_{S}$ to $\Delta$ is absolutely continuous with respect to the Lebesgue measure on $\Delta$

(iv) the local measures $p_{1, a}$ and $p_{2, b}$ are rotation invariant, i.e.

$p_{1, a+\delta}\left(s_{1}+\delta\right)=p_{1, a}\left(s_{1}\right) \quad ; \quad p_{2, b+\delta}\left(s_{2}+\delta\right)=p_{2, b}\left(s_{2}\right) \quad ; \quad \forall \delta \in \mathbf{R}$

(v) the local measures $p_{1, a}$ and $p_{2, b}$ are twice continuously differentiable, 
Then the probability measure $d R_{a, b}\left(s_{1}, s_{2}\right)$, defined by (11), must have either the form

$$
d R_{a, b}\left(s_{1}, s_{2}\right)=\delta\left(s_{1}-s_{2}\right) d s_{1} d s_{2} \frac{1}{4}\left|\cos \left(s_{1}-a\right)\right|
$$

or the form

$$
d R_{a, b}\left(s_{1}, s_{2}\right)=\delta\left(s_{1}-s_{2}\right) d s_{1} d s_{2} \frac{1}{4}\left|\cos \left(s_{2}-b\right)\right| .
$$

Proof. Because of rotation invariance

$$
\begin{aligned}
& p_{1, a}\left(s_{1}\right)=p_{1,0}\left(s_{1}-a\right)=: p_{1}\left(s_{1}-a\right) \\
& p_{2, b}\left(s_{2}\right)=p_{2,0}\left(s_{2}-b\right)=: p_{2}\left(s_{2}-b\right) .
\end{aligned}
$$

Using the concentration of $P_{S}$ on the diagonal, we have

$$
d R_{a, b}\left(s_{1}, s_{2}\right)=\rho\left(s_{1}\right) p_{1}\left(s_{1}-a\right) p_{2}\left(s_{2}-b\right) \delta\left(s_{1}-s_{2}\right) d s_{1} d s_{2} .
$$

For $a$ and $b$ satisfying $0 \leq b-a \leq \pi, I_{a} \cap I_{b}=[-\pi / 2+b, a+\pi / 2)$, and therefore

$$
R_{a, b}\left(I_{a} \times I_{b}\right)=\int_{-\pi / 2+b}^{a+\pi / 2} d s_{1} \rho\left(s_{1}\right) p_{1}\left(s_{1}-a\right) p_{2}\left(s_{1}-b\right)
$$

From (12) we deduce that,

$$
\frac{1}{4}(1+\cos (b-a))=R_{a, b}\left(I_{a} \times I_{b}\right)=\int_{-\pi / 2+b}^{a+\pi / 2} d s_{1} \rho\left(s_{1}\right) p_{1}\left(s_{1}-a\right) p_{2}\left(s_{1}-b\right)
$$

Differentiating this with respect to $b$, we find

$$
\begin{gathered}
-\frac{1}{4} \sin (b-a)=-\rho(b-\pi / 2) p_{1}(b-a-\pi / 2) p_{2}(-\pi / 2) \\
+\int_{b-\pi / 2}^{a+\pi / 2} d s_{1} \rho\left(s_{1}\right) p_{1}\left(s_{1}-a\right) p_{2}^{\prime}\left(s_{1}-b\right)
\end{gathered}
$$


Putting $b=a+\pi$, we obtain

$$
0=\rho(a+\pi / 2) p_{1}(\pi / 2) p_{2}(-\pi / 2)
$$

Since $\rho$ is a probability density, $\rho(a+\pi / 2)$ cannot vanish for all $a$. Since $a$ is arbitrary it follows that

$$
p_{1}(\pi / 2)=0 \text { or } p_{2}(-\pi / 2)=0
$$

Let us assume that $p_{1}(\pi / 2)=0$.

Differentiating again with respect to $b$ and putting $b=a+\pi$, we obtain

$$
\frac{1}{4}=-\rho(a+\pi / 2) p_{1}^{\prime}(\pi / 2) p_{2}(-\pi / 2)
$$

From this we can see

$$
p_{1}^{\prime}(\pi / 2) \neq 0 \text { and } p_{2}(-\pi / 2) \neq 0
$$

and therefore

$$
\rho(a+\pi / 2)=1 /\left(4 p_{1}^{\prime}(\pi / 2) p_{2}(-\pi / 2)\right)=(\text { const. })
$$

since $a$ is arbitrary we deduce that $\rho\left(s_{1}\right)=c$. Since $I_{a} \cap J_{b}=$ $[-\pi / 2+a,-\pi / 2+b),(12)$ implies that $\frac{1}{4}(1-\cos (b-a))=R_{a, b}\left(I_{a} \times J_{b}\right)=c \int_{-\pi / 2+b}^{-\pi / 2+a} d s_{1} p_{1}\left(s_{1}-a\right) p_{2}\left(s_{1}-b\right)$

Differentiating this with respect to $b$, we have

$$
\frac{1}{4} \sin (b-a)=-c p_{1}(b-a-\pi / 2) p_{2}(-\pi / 2)+c \int_{-\pi / 2+b}^{-\pi / 2+a} d s_{1} p_{1}\left(s_{1}-a\right) p_{2}^{\prime}\left(s_{1}-b\right)
$$

Putting $b=a$, we obtain

$$
0=-c p_{1}(-\pi / 2) p_{2}(-\pi / 2)
$$


Since $p_{2}(-\pi / 2) \neq 0$ it follows that:

$$
p_{1}(-\pi / 2)=0
$$

Since $\left(I_{a} \cap J_{b}\right) \cup\left(I_{a} \cap I_{b}\right)=[-\pi / 2+a, a+\pi / 2)$, by (12) we have

$$
\frac{1}{2}=R_{a, b}\left(I_{a} \times J_{b} \cup I_{a} \times I_{b}\right)=c \int_{-\pi / 2+a}^{a+\pi / 2} d s_{1} p_{1}\left(s_{1}-a\right) p_{2}\left(s_{1}-b\right)
$$

Changing variable with $s=s_{1}-a$, we obtain

$$
\frac{1}{2}=c \int_{-\pi / 2}^{\pi / 2} d s p_{1}(s) p_{2}(s-b+a)
$$

In the same way, for $\pi \leq b-a \leq 2 \pi$ one has,

$$
I_{a} \cap I_{b}=[-\pi / 2+a,-3 \pi / 2+b) \quad ; \quad I_{a} \cap J_{b}=[-3 \pi / 2+b, a+\pi / 2)
$$

Therefore we have

$$
\begin{gathered}
\frac{1}{2}=R_{a, b}\left(I_{a} \times I_{b} \cup I_{a} \times J_{b}\right) \\
=c \int_{-\pi / 2+a}^{a+\pi / 2} d s_{1} p_{1}\left(s_{1}-a\right) p_{2}\left(s_{1}-b\right)=c \int_{-\pi / 2}^{\pi / 2} d s p_{1}(s) p_{2}(s-b+a)
\end{gathered}
$$

Since $p_{1}$ is continuous and $a$ and $b$ are arbitrary, we conclude that

$$
\begin{array}{r}
p_{2}(s)=\text { const. }=: c_{2} \text {. Thus by renaming } \\
\widetilde{p}_{1}\left(s_{1}\right):=c p_{1}\left(s_{1}\right) c_{2}
\end{array}
$$

we find

$$
d R_{a, b}\left(s_{1}, s_{2}\right)=\widetilde{p}_{1}\left(s_{1}-a\right) \delta\left(s_{1}-s_{2}\right) d s_{1} d s_{2} .
$$

Our remaining task is to determine the form of $\widetilde{p}_{1}$. 
For $a$ and $b$ satisfying $0 \leq b-a \leq \pi$ the above calculations lead to

$$
-\frac{1}{4} \sin (b-a)=-\widetilde{p}_{1}(-\pi / 2+b-a)
$$

By putting $s:=b-\pi / 2$, we obtain

$$
\widetilde{p}_{1}(s-a)=\frac{1}{4} \cos (s-a) \text { for }-\pi / 2 \leq s-a \leq \pi / 2
$$

Therefore

$$
\widetilde{p}_{1}(s-a)=\frac{1}{4}|\cos (s-a)|,-\pi / 2 \leq s-a \leq \pi / 2 .
$$

Since $J_{a} \cap I_{b}=[a+\pi / 2, b+\pi / 2)$,

$$
\frac{1}{4}(1-\cos (b-a))=R_{a, b}\left(J_{a} \times I_{b}\right)=\int_{a+\pi / 2}^{b+\pi / 2} d s_{1} \widetilde{p}_{1}\left(s_{1}-a\right) .
$$

By differentiating this with respect to $b$ we have

$$
\frac{1}{4} \sin (b-a)=\widetilde{p}_{1}(b+\pi / 2-a) .
$$

By putting $s=b+\pi / 2, \widetilde{p}_{1}(s-a)=\frac{1}{4} \sin (s-a-\pi / 2)=$ $-\frac{1}{4} \cos (s-a)$ for $\pi / 2 \leq s-a \leq 3 \pi / 2$. Therefore

$$
\widetilde{p}_{1}(s-a)=\frac{1}{4}|\cos (s-a)|, \pi / 2 \leq s-a \leq 3 \pi / 2 .
$$

Accordingly,

$$
d R_{a, b}\left(s_{1}, s_{2}\right)=\delta\left(s_{1}-s_{2}\right) d s_{1} d s_{2} \frac{1}{4}\left|\cos \left(s_{1}-a\right)\right| .
$$

If we assume that $p_{2}(-\pi / 2)=0$ instead of $p_{1}(\pi / 2)=0$, then in the same way we obtain

$$
d R_{a, b}\left(s_{1}, s_{2}\right)=\delta\left(s_{1}-s_{2}\right) d s_{1} d s_{2} \frac{1}{4}\left|\cos \left(s_{2}-b\right)\right|
$$




\section{Summarizing}

The genericity assumptions used in the proof of the above uniqueness theorem are the following:

- (i) The local causal structure of the probability measure

- (i) The condition of statistical pre-determination

- (ii) The rotation invariance of the densities describing the local apparata

- (iii) The twice continuous differentiability of these densities

- (iv) The absolute continuity of the source measure with respect to the Lebesgue measure

While conditions (i) and (ii) have a natural physical interpretation, we don't see a natural physical justification for conditions (iii) and (iv).

For example at the moment we have no reasons to exclude the possibility of reproducing the EPR correlations with a source measure having a fractal support.

Therefore it would be interesting to know if, by dropping some of these assumptions, the uniqueness result continues to be true. This problem will be the object of further investigations.

\section{Local, causal measurements}

von Neumann's theory of measurement did not incorporate the locality requirement, which is essential in the discussion of EPR type experiments.

In the following we will briefly outline the main properties of a theory of local, causal measurements in a classical nonrelativistic context. We refer to the survey paper [5] for the quantum formulation and additional information. 
We consider a composite system made up of two subsystems, called 'particles' in the following, and denoted with the symbols 1 and 2 respectively. Their 'configuration' (or 'phase') spaces will be denoted by $S_{1}$ and $S_{2}$ respectively.

The two systems are spatially separated so that the mutual interactions between them can be neglected. Each system interacts locally with a measurement apparatus, i.e. system 1 with apparatus $m_{1}$ and system 2 with apparatus $m_{2}$. The configuration spaces of the measurement apparata will be denoted by $M_{1}$ and $M_{2}$ respectively. We use the indices $a, b, \ldots \in I$ to represent settings of the measurement apparata.

Definition 3 (Ref. [2], Definition 6.) A probability measure $P_{a, b}$ on

$S_{1} \times S_{2} \times M_{1} \times M_{2}$ is called extremal, local and causal (extremal$L C$, shortly) if it has the form

$$
d P_{a, b}\left(s_{1}, s_{2}, \lambda_{1}, \lambda_{2}\right)=d P_{S}\left(s_{1}, s_{2}\right) P_{1, a}\left(d \lambda_{1} ; s_{1}\right) P_{2, b}\left(d \lambda_{2} ; s_{2}\right)
$$

where $P_{S}$ is a probability measure on $S_{1} \times S_{2}$; for all $s_{1} \in$ $S_{1}, P_{1, a}\left(\cdot ; s_{1}\right)$ is a positive measure on $M_{1}$; for all $s_{2} \in S_{2}$, $P_{2, b}\left(\cdot ; s_{2}\right)$ is a positive measure on $M_{2}$.

It is called local and causal ( $L C$, shortly) if it is a convex combination of extremal-LC measures, i.e. if it has the form $d P_{a, b}\left(s_{1}, s_{2}, \lambda_{1}, \lambda_{2}\right)=d P_{S}\left(s_{1}, s_{2}\right) \int_{X} q(d x) P_{1, a}\left(d \lambda_{1} ; s_{1} ; x\right) P_{2, b}\left(d \lambda_{2} ; s_{2} ; x\right)$

where $P_{S}, P_{1, a}\left(\cdot ; s_{1} ; x\right), P_{2, b}\left(\cdot ; s_{2} ; x\right)$ are as above and $(X, q)$ is a probability space.

Remark (1) We will see in Theorem (2) that, under generic regularity conditions, there exist only two extremal-LC measures and they attribute an asymmetric role to the two particles 
1 and 2. Non extremal-LC measures are useful to build statistical models in which the two particles appear in a symmetric way.

\section{Remark (2)}

All experimentally measured statistics depend on:

- a preparation,

- an observable

- a dynamics.

In classical physics the mathematical model of an experimental preparation is given by a probability measure (a quantum state in quantum physics). The extreme case of Dirac $\delta$-measures correspond to exact theories.

In EPR type experiments the experimental preparation is localized in different space-time regions:

- the emission time and the interaction times

- the emission region and the local measurement regions

The time separation involves causality: at the emission time, the type of interation that will be met at the measurement time is unknown. Therefore the statistics at the source cannot depend on this interaction. This causality condition is reflected in the factorization condition $P_{a, b}=P_{S} \cdot Q_{M_{1, a} \times M_{2, b}}$, where $Q_{M_{1, a} \times M_{2, b}}\left(\cdot ; s_{1}, s_{2}\right)$ is a positive measure on $M_{1} \times M_{2}$.

The space separation involves locality: the statistics of the local measurements should be mutually independent except for possible conservation laws realized at the emission time (pre-determination).

Locality is reflected in the fact that $Q_{M_{1, a} \times M_{2, b}}\left(\cdot ; s_{1}, s_{2}\right)$ is factorized as $P_{1, a}\left(\cdot ; s_{1}\right) P_{2, b}\left(\cdot ; s_{2}\right)$

Remark. 
Note that it is not required that $P_{1, a}\left(\cdot ; s_{1}\right)$ and $P_{2, b}\left(\cdot ; s_{2}\right)$ are conditional probability measures. We will come back to this point in section ().

Now we introduce the phase (or configuration) spaces of our systems: for the moment we introduce them in an abstract way, without specifying their structure (this might include position, momentum, spin, ..., and possibly othe hidden parameters). We only assume, to simplify the mathematical description, that they are compact Hausdorff spaces:

- the configuration space $S_{1}$ of the particle 1 ,

- the configuration space $S_{2}$ of the particle 2 ,

- the configuration space $M_{1}$ of the measurement apparatus for the particle 1 ,

- the configuration space $M_{2}$ of the measurement apparatus for the particle 2 .

In terms of these we define the configuration spaces for the composite systems:

$$
\begin{gathered}
S:=S_{1} \times S_{2} ; M:=M_{1} \times M_{2} ; \Omega_{1}:=S_{1} \times M_{1} ; \Omega_{2}:=S_{2} \times M_{2} \\
\Omega:=\Omega_{1} \times \Omega_{2}=S_{1} \times M_{1} \times S_{2} \times M_{2}=S_{1} \times S_{2} \times M_{1} \times M_{2}(18)
\end{gathered}
$$

We will use the following notations: $\operatorname{Meas}(\Omega)$ denotes the set of all regular, signed, finite Borel measures on $\Omega$. $\langle\operatorname{Meas}(\Omega), C(\Omega)\rangle$ denotes the duality, between $C(\Omega)$ and $\operatorname{Meas}(\Omega)=C(\Omega)^{*}$, given by the integral:

$$
\langle P, f\rangle:=\int_{\Omega} f(\omega) P(d \omega)
$$

$\mathrm{Meas}_{+}(\Omega)$ and $\operatorname{Prob}(\Omega)$ denote the set of all positive measures and the set of all probability measures in Meas $(\Omega)$ respectively. 
Any LC measure on $S_{1} \times S_{2} \times M_{1} \times M_{2}$, of the form (16), can be written in the following functional form:

$$
P_{a, b}:=P_{S} \circ\left(\bar{P}_{1, a} \otimes \bar{P}_{2, b}\right) \in\left(C\left(\Omega_{1}\right) \otimes C\left(\Omega_{2}\right)\right)^{*}=C\left(\Omega_{1} \times \Omega_{2}\right)^{*}
$$

where, for $j=1,2$ and $x=a, b$, the linear maps $\bar{P}_{j, x}: C\left(\Omega_{j}\right)=C\left(S_{j} \times M_{j}\right) \rightarrow C\left(S_{j}\right) \subseteq C\left(\Omega_{j}\right)$ are defined by

$$
\bar{P}_{j, x}(f)\left(s_{j}\right):=\int_{M_{j}} f\left(s_{j}, \lambda_{j}\right) d P_{j, x}\left(\lambda_{j} ; s_{j}\right)
$$

for each $f \in C\left(S_{j} \times M_{j}\right)$.

\section{Trivial extremal LC probability measures}

The notion of trivial $L C$ probability measure is crucial for the EPR-chameleon models.

Definition 4 (Ref. [2], Definition 7.) An extremal LC probability measure on the space $S_{1} \times S_{2} \times M_{1} \times M_{2}$

$$
d P_{a, b}\left(s_{1}, s_{2}, \lambda_{1}, \lambda_{2}\right)=d P_{S}\left(s_{1}, s_{2}\right) d P_{1, a}\left(\lambda_{1} ; s_{1}\right) d P_{2, b}\left(\lambda_{2} ; s_{2}\right)
$$

is called trivial if, in the notation (20), $\forall a, b \in I$ the map

$$
\bar{P}_{1, a} \otimes \bar{P}_{2, b}: C\left(\Omega_{1} \times \Omega_{2}\right) \rightarrow C\left(S_{1} \times S_{2}\right)
$$

is a $P_{S}-$ conditional expectation i.e.

$$
\bar{P}_{1, a}\left(1_{1}\right)\left(s_{1}\right) \bar{P}_{2, b}\left(1_{2}\right)\left(s_{2}\right) \equiv 1 \quad, \quad P_{S^{-}} \text {a.e. }
$$

Denoting

$$
p_{1, a}\left(s_{1}\right):=\bar{P}_{1, a}\left(1_{1}\right)\left(s_{1}\right)=\int_{M_{1}} d P_{1, a}\left(\lambda_{1} ; s_{1}\right)
$$




$$
p_{2, b}\left(s_{2}\right):=\bar{P}_{2, b}\left(1_{2}\right)\left(s_{2}\right)=\int_{M_{2}} d P_{2, b}\left(\lambda_{2} ; s_{2}\right)
$$

condition (21) becomes equivalent to:

$$
p_{1, a}\left(s_{1}\right) p_{2, b}\left(s_{2}\right)=1 \quad, \quad P_{S^{-} \text {-a.e. }}
$$

If a LC measure is trivial, then there exists a positive real number $c$ such that

$$
p_{1, a}\left(s_{1}\right)=c, p_{2, b}\left(s_{2}\right)=\frac{1}{c} \quad, P_{S^{-}} \text {a.e. }
$$

By redefining $P_{1, a}^{\prime}:=(1 / c) P_{1, a}, P_{2, b}^{\prime}:=c P_{2, b}$, we can assume without loss of generality that

$$
p_{1, a}\left(s_{1}\right)=1, p_{2, b}\left(s_{2}\right)=1 \quad, P_{S^{-}} \text {a.e. }
$$

The following proposition shows that triviality implies Bell type inequalities.

Proposition 1 Let $I$ be any index set and let $P_{a, b}(a, b \in I)$ be a family of trivial LC probability measures on the space $\Omega$ defined by (18) and let $S_{a}^{(1)}, S_{b}^{(2)}: \Omega \rightarrow[-1,1](a, b \in I)$ be a family of random variables satisfying the locality condition

$$
\begin{gathered}
S_{a}^{(1)}\left(\omega_{1}, \omega_{2}\right)=S_{a}^{(1)}\left(\omega_{1}\right) ; S_{b}^{(2)}\left(\omega_{1}, \omega_{2}\right)=S_{b}^{(2)}\left(\omega_{2}\right) ; \\
\left(\omega_{1}, \omega_{2}\right) \in \Omega=\Omega_{1} \times \Omega_{2}, a, b \in I
\end{gathered}
$$

For any ordered pair $(a, b)$, with $a, b \in I$, define the $P_{a, b}-$ pair correlation of the pair $\left(S_{a}^{(1)}, S_{b}^{(2)}\right)$ in the usual way, i.e.

$$
C(a, b):=\int S_{a}^{(1)} S_{b}^{(2)} d P_{a, b}
$$

(Notice that the probability measure depends on the pair $(a, b)$ ). Then the family of pair correlations $\{C(a, b): a, b \in I\}$ satisfies the Bell inequality in the CHSH form (but not necessarily in the original Bell form). 
Proof. See Ref. [1, 3].

Remark (1). The above theorem provides an example of a context dependent family of pair correlations which must satisfy Bell's inequality. This proves that the contextuality condition alone is not sufficient to guarantee the violation of this inequality.

Remark (2). The locality condition (25) is necessary only because of the assumption the probability measures in the family $\left.\left\{P_{a, b}: a, b \in I\right\}\right)$ depend on the pair $(a, b)$ (contextuality)). As shown in [6], Theorem (3), in the standard context considered in the literature (a single probability measure and no chameleon dynamics) this assumption is not necessary.

Remark (3). To be a trivial LC measure is a sufficient, but not necessary condition to satisfy Bell's inequality: there are examples of nontrivial LC measures which do not violate Bell's inequality.

Recall that, if $\Omega, S$ are topological spaces, a linear map $\mathcal{T}^{*}: C(\Omega) \rightarrow C(S)$ is called a Markov operator if it is positivity preserving $\left(f \geq 0 \Rightarrow \mathcal{T}^{*}(f) \geq 0, f \in \mathcal{C}(\Omega)\right)$ and identity preserving:

$$
\mathcal{T}^{*}\left(1_{\Omega}\right)=1_{S}
$$

If on $S$ there is a probability measure $P_{S}$ and $\mathcal{T}^{*}$ satisfies the weaker conditions

$$
\begin{gathered}
f \geq 0 \Rightarrow \mathcal{T}^{*}(f) \geq 0 ; \quad P_{S^{-}} \text {a.e. } f \in \mathcal{C}(\Omega) \\
\mathcal{T}^{*}\left(1_{\Omega}\right)=1_{S}, \quad P_{S^{-} \text {-a.e. }}
\end{gathered}
$$

we call it a $P_{S}$-Markov operator. Now let

$$
\Omega=\Omega_{1} \times \Omega_{2} ; \quad S=S_{1} \times S_{2}
$$


Lemma 1 For $j=1,2$, let $\mathcal{T}_{j}^{*}: C\left(\Omega_{j}\right) \rightarrow C\left(\Omega_{j}\right)$ be a positivity preserving linear operator. The following conditions are equivalent:

$$
\bar{P}_{1, a}\left(\mathcal{T}_{1}^{*}(1)\right) \bar{P}_{1, b}\left(\mathcal{T}_{2}^{*}(1)\right)=1 ; P_{S}-\text { a.e. }
$$

there exists a constant $c>0$ such that

$$
\bar{P}_{1, a}\left(c \mathcal{T}_{1}^{*}(1)\right)=\bar{P}_{1, b}\left(\mathcal{T}_{2}^{*}(1) / c\right)=1 ; P_{S} \text {-a.e. }
$$

Proof. It is clear that $(27) \Rightarrow(26)$. Let us prove the converse implication. If (26) holds, then

$$
P_{S} \circ\left(\left[\bar{P}_{1, a} \circ \mathcal{T}_{1}^{*}\right] \otimes\left[\bar{P}_{2, b} \circ \mathcal{T}_{2}^{*}\right]\right)
$$

is a trivial measure. Therefore, by the remark below Definition (4) there exists a constant $c>0$ such that

$c \bar{P}_{1, a}\left(\mathcal{T}_{1}^{*}(1)\right)\left(s_{1}\right)=\frac{1}{c} \bar{P}_{2, b}\left(\mathcal{T}_{2}^{*}(1)\right)\left(s_{2}\right)=1 \quad ; \quad P_{S}-\forall\left(s_{1}, s_{2}\right) \in S_{1} \times S_{2}$ and this is (27).

Definition 5 A linear positive operator $\mathcal{T}_{1}^{*} \otimes \mathcal{T}_{2}^{*}: C\left(\Omega_{1} \times \Omega_{2}\right) \rightarrow$ $C\left(\Omega_{1} \times \Omega_{2}\right)$ (or equivalently its dual $\mathcal{T}_{1} \otimes \mathcal{T}_{2}$, acting on measures), which satisfies the conditions of Lemma (1), will be called a $P_{a, b}{ }^{-}$ Markovian operator. In such a case, by absorbing the constants c, $1 / c$ in the definition of $\mathcal{T}_{1}^{*}$ and $\mathcal{T}_{2}^{*}$, one can always assume that they are equal to 1.

Notice that any Markov operator from $C\left(\Omega_{1} \times \Omega_{2}\right)$ to $C\left(\Omega_{1} \times\right.$ $\left.\Omega_{2}\right)$ is $P_{a, b}-$ Markovian for any $P_{a, b}$. 
Theorem 3 Let, for $j=1,2, \mathcal{T}_{j}$ be a linear mapping of $\operatorname{Meas}_{+}\left(\Omega_{j}\right)$ into Meas $_{+}\left(\Omega_{j}\right)$ such that $\mathcal{T}_{j}^{*}: C\left(\Omega_{j}\right) \rightarrow C\left(\Omega_{j}\right)$ and let

$$
P_{a, b}=P_{S} \circ\left(\bar{P}_{1, a} \otimes \bar{P}_{2, b}\right) \in \operatorname{Prob}\left(\Omega_{1} \times \Omega_{2}\right)
$$

be any trivial $L C$ measure. Then if $\mathcal{T}_{1, a} \otimes \mathcal{T}_{2, b}$ is a $P_{a, b}$-Markovian operator, $\left(\mathcal{T}_{1, a} \otimes \mathcal{T}_{2, b}\right)\left(P_{a, b}\right)$ is a trivial LC measure.

In particular, if $\mathcal{T}_{1, a} \otimes \mathcal{T}_{2, b}$ is a Markov operator, it maps trivial $L C$ measures into trivial $L C$ measures.

Proof. The functional form of $\left(\mathcal{T}_{1, a} \otimes \mathcal{T}_{2, b}\right)\left(P_{a, b}\right)$ is:

$$
\left(\mathcal{T}_{1, a} \otimes \mathcal{T}_{2, b}\right)\left(P_{a, b}\right)=P_{S} \circ\left(\bar{P}_{1, a} \circ \mathcal{T}_{1, a}^{*} \otimes \bar{P}_{2, b} \circ \mathcal{T}_{2, b}^{*}\right) .
$$

Condition (27) (with $c=1$ ) is equivalent to

$$
\bar{P}_{1, a}\left(\mathcal{T}_{1}^{*}(1)\right)=\bar{P}_{2, b}\left(\mathcal{T}_{2}^{*}(1)\right)=1 ; P_{S^{-} \text {a.e. }}
$$

which is equivalent to the triviality of $\left(\mathcal{T}_{1, a} \otimes \mathcal{T}_{2, b}\right)\left(P_{a, b}\right)$.

Corollary 1 Any local reversible dynamics induces a mapping which maps a nontrivial (resp. trivial) LC measure into a nontrivial (resp. trivial) LC measure.

Proof. The statement about trivial LC measures follows from Theorem (3).

Let $\mu$ be a nontrivial LC measure and $T$ be a reversible measurable transformation of $S_{1} \times M_{1} \times S_{2} \times M_{2}$ into itself. Suppose by contradiction that $\nu:=\mu \circ T$ is trivial. The linear mapping $\mathcal{T}$ induced by $T$ is a Markov operator satisfying $\mu=\mathcal{T}(\nu):=\nu \circ T^{-1}$. Its inverse is also a Markov operator satisfying $\nu=\mathcal{T}^{-1}(\mu):=\mu \circ T$. 
But if $T$ is local i.e. of the form $T=T_{1} \times T_{2}$ for some $T_{1}: S_{1} \times M_{1} \rightarrow S_{1} \times M_{1}$ and $T_{2}: S_{2} \times M_{2} \rightarrow S_{2} \times M_{2}$, then $\mathcal{T}=\mathcal{T}_{1} \otimes \mathcal{T}_{2}$ where $\mathcal{T}_{1}$ and $\mathcal{T}_{2}$ are Markov operators. By the remark after Definition (5) this contradicts Theorem (3).

\section{Bell implicitly assumes triviality}

In "Bertlmann's sock and the nature of reality" [7] Bell requires the condition

$$
P(A, B \mid a, b, \lambda)=P_{1}(A \mid a, \lambda) P_{2}(B \mid b, \lambda) .
$$

and argues that it is reasonable to expect that $P_{1}$ and $P_{2}$ are conditional probability distributions.

Given the following correspondence between the notations in Bell's argument and those of Definition (4):

$\lambda \leftrightarrow\left(s_{1}, s_{2}\right), P_{1}(A \mid a, \lambda) \leftrightarrow \bar{P}_{1, a} \circ \mathcal{T}_{1, a}^{*}, P_{2}(B \mid b, \lambda) \leftrightarrow \bar{P}_{2, b} \circ \mathcal{T}_{2, b}^{*}$

we see that Bell implicitly assumes triviality of the LC measure.

This assumption is equivalent to postulate that the stochastic processes given by the observables of the two systems at the final (measurement) time are conditionally independent given the source.

However such an assumption is physically and probabilistically unwarranted whenever there are constraints (e.g. conservation laws) which are determined both at the source and at the local measurement sites. For example, if $S_{a}^{(1)}, S_{a}^{(2)}$ are random variables that depend both on the source and the apparatus variables and if we know that, at the time of measurement, they 
must satisfy the constraint:

$$
S_{a}^{(1)}\left(s_{1}, \lambda_{1}\right)+S_{a}^{(2)}\left(s_{2}, \lambda_{2}\right)=0
$$

then surely they are not conditionally independent given the source variables $\left(s_{1}, s_{2}\right)$ : here the difference between a preexistent property and a property pre-determined as a response to a local interaction (the if ... then ... scheme) is essential.

Since this is precisely the situation in the EPR type experiments, we see that Bell's implicit assumption is not justified in this case.

The class of LC measures is the disjoint union of its two subclasses, of the trivial and the nontrivial LC measures. Corollary (1) implies that a local reversible dynamics induces a map of each of these classes into itself.

Since a local measurement process is described by a local Markov operator, it follows that the initial probability measure,in the EPR-chameleon model, must be nontrivial otherwise one could not have violation of Bell's inequality.

The above discussion proves that the nontriviality of the initial LC measure is precisely what is required by the physical context of the EPR type experiments where locality and causality have to be combined with the existence of constraints (conservation laws). Conversely, Bell's implicit assumption of the triviality of the initial measure, being equivalent to conditional independence given the source, puts the locality and causality requirement in contradiction with the conservation laws.

The dependence of the initial state on the pair $(a, b)$ is not a non-local requirement because, for adaptive dynamical systems, the ensamble to which the individual systems belong is not defined at the emission time but at the time in which the interaction with the local instruments begins to take place. 
In other words, since for such systems the measurement locally affects the dynamics, the natural notion of classical statistical state is not, as for passive systems, a single probability measure (corresponding to the fact that the state will be independent of the measurement), but a family of probability measures - one for each possible measurement (corresponding to the fact that the state will be determined by the response to a measurement which is not known at the initial time).

The EPR-chameleon model shows that all this is perfectly compatible with a local, deterministic, reversible dynamics.

\section{Empirical Correlations of pairs of distant particles}

The same term 'pair correlation', when referred to pairs of distant particles is often used to describe two completely different experimental procedures. Below we discuss these experimental differences [3].

\subsection{Standard correlations}

The term standard correlation is used when the following physical conditions are verified:

1) The total number $N$ of emitted pairs is exactly known.

2) The trajectory of each pair can be followed without disturbance so that, at each time $t$, the experimenters know exactly to which of the $N$ pairs their measurement is referred. This property will be called distinguishability. 
3) The observable $\left(f_{1}, f_{2}\right)$ is measured on each pair of the ensemble. The result of the measurement of $\left(f_{1}, f_{2}\right)$ on the $j$ th pair will be denoted by

$$
\left(f_{1, j}, f_{2, j}\right)
$$

the measurement itself will be denoted by $M_{j}$.

Under these conditions the following definition makes sense.

Definition 6 The empirical correlation between the pair of observables $\left(f_{1}, f_{2}\right)$, relative to the sequence of measurements $M=$ $\left(M_{j}\right)$ on the ensemble $\left\{\left(1_{j}, 2_{j}\right): j=1, \ldots, N\right\}$ is

$$
\left\langle f_{1} \cdot f_{2}\right\rangle_{M}:=\frac{1}{N} \sum_{j=1}^{N} f_{1, j} f_{2, j} .
$$

We further specify our context of standard correlations as follows.

4) Each measurement $M_{j}$ is specified by a time

$$
t_{j}^{\prime}:=t_{j}+T,
$$

where $T$ is independent of $j, t_{j}$ is the emission time for the pair $\left(1_{j}, 2_{j}\right)$.

5) The result of the $j$ th measurement does not depend on the interval $\left[t_{j}, t_{j}+T\right]$ but only on $T$ (time homogeneity).

Under these conditions the correlations (29) are interpreted as the correlations of $\left(f_{1}, f_{2}\right)$ at time $T$ and $T$ is interpreted as the final time of the single measurement. 


\subsection{Correlations of distant pairs}

Suppose that the measurement protocol is the following.

(DP1) It is known that each pair is emitted simultaneously, but the experimenters do not know precisely when the pair is emitted.

(DP2) The experimenters cannot follow the trajectory of each particle, but only register the result of a measurement at time $t$ (indistinguishability).

(DP3) The experimenters have synchronized clocks, so the time $t$ is the same for both.

(DP4) The experimenters do not know the total number of emitted particles.

(DP5) The experimenters cannot postulate that, if a particle of a pair reaches one of them, then the other particle reaches the other experimenters.

Conditions (4) and (5) of the previous section are still meaningful because they are referred to single particles. However condition (3) is meaningless because of indistinguishability. Moreover the $N$, in formula (29) is unknown. In a situation described by the above conditions we speak of correlations of distant particles.

In conclusion: under the above described physical conditions, the definition of standard correlations is meaningless and a new one is needed.

Definition 7 The protocol to define correlations of distant particles is the following: 
(CDP1) The experimenter $X, X \in\{1,2\}$ performs measurements on $M_{X}$ particles and records

- the time $t_{X, j}^{\prime}$ of the $j$ th measurement

- the value $f_{X, j}$ of the measured observable $f_{X}$

for $\forall j \in\left\{1, \ldots, M_{X}\right\}$.

(CDP2) The two experimenters exchange the sequences

$$
\left(\left(t_{1, j}^{\prime}, f_{1, j}\right): j=1, \ldots, M_{1}\right) \text { and }\left(\left(t_{2, j}^{\prime}, f_{2, j}\right): j=1, \ldots, M_{2}\right) .
$$

(CDP3) Each experimenter extracts the sequences

$$
\left(f_{1, h}^{\prime}: h=1, \ldots, M_{f_{1} f_{2}}\right) \text { and }\left(f_{2, h}^{\prime}: h=1, \ldots, M_{f_{1} f_{2}}\right),
$$

where

$\left\{s_{h}: h \in\left\{1, \ldots, M_{f_{1} f_{2}}\right\}\right\}:=\left\{t_{1, j}^{\prime}: j \in\left\{1, \ldots, M_{1}\right\}\right\} \cap\left\{t_{2, j}^{\prime}: j \in\left\{1, \ldots, M_{2}\right.\right.$

and

$$
f_{X, h}^{\prime}:=f_{X, j}, \text { if } s_{h}=t_{X, j}^{\prime} \quad(X=1,2) .
$$

(CDP4) The empirical correlations of distant pairs are defined by

$$
\left\langle f_{1} f_{2}\right\rangle_{D P}:=\frac{1}{M_{f_{1}, f_{2}}} \sum_{h=1}^{M_{f_{1}, f_{2}}} f_{1, h}^{\prime} f_{2, h}^{\prime} .
$$

In other words: by definition, correlation of distant pairs means conditioned correlations on coincidences.

Practically the totality of the EPR type experiments follows the protocol described in Definition (7).

As far as experiments are performed under this protocol (Definition (7)), it is possible to take the Bell's fifth position named by Gill: "a decisive experiment cannot be done" [8]. 


\section{Riferimenti bibliografici}

[1] L. Accardi, K. Imafuku, and M. Regoli, Infinite Dimensional Analysis, Quantum Probability and Related Topics, 5, 1-20 (2002) quant-ph/0112067, Volterra Preprint N. 494 December (2001) N. 494.

[2] L. Accardi, K. Imafuku, and M. Regoli, "Adaptive dynamical systems and the EPR-chameleon experiment, in Foundations of Probability and Physics - 2, edited by A. Khrennikov, Mathematical Modeling in Physics, Engineering and Cognitive Science 5, Växjö University Press, Växjö, Sweden-2002, 2003, pp. 11-36.

[3] L. Accardi, and S. Uchiyama, arXiv:quant-ph 0706.1813 (2007). submitted to: IDA-QP (Infinite Dimensional Analysis, Quantum Probability and Related Topics) 14 June (2007)

[4] Luigi Accardi, Andrei Khrennikov: Chameleon effect, the range of values Hypothesis and reproducing the EPR-Bohm correlations, Foundations of probability and physics-4 AIP Proceedings 889 (2006) 21-29

[5] L. Accardi, "Could we now convince Einstein?, in: Proceedings of the conference: Quantum theory: reconsideration of foundations-3, Växjö, Sweden 6-11 june (2005), Andrei Khrennikov (ed.), American Institute of Physics, AIP Conference Proceedings 810 (2006) 2-18, Melville, New York, ISBN 0-7354-0301-5.

[6] L. Accardi, and M. Regoli, Volterra Preprint N. 427; arXiv: quant-ph/0007005 (2000). 
[7] J. S. Bell, Speakable and Unspeakable in Quantum Mechanics, Cambridge University Press, Cambridge, 1987.

[8] R. D. Gill, "Time, Finite Statistics, and Bell's Fifth Position, in Foundations of Probability and Physics - 2, edited by A. Khrennikov, Mathematical Modeling in Physics, Engineering and Cognitive Science 5, Växjö University Press, Växjö, Sweden-2002, 2003, pp. 179-206. 